\title{
Evaluation of Postoperative Chemotherapy Duration of Capecitabine-Alone Regimen for Surgery Treated Elderly Colon Cancer Patients : A Cohort Study
}

Weiwei Chen ( $\nabla$ weiweichen@gmc.edu.cn)

Guizhou Medical University https://orcid.org/0000-0002-8275-9162

Hongmin Dong

The Affiliated Hospital of Guizhou Medical University

Gang Wang

The Affiliated Hospital of Guizhou Medical University

Juan Chen

Guizhou Medical University

Wenling Wang

The Affiliated Hospital of Guizhou Medical University

\section{Research Article}

Keywords: Postoperative chemotherapy, Capecitabine, Colon cancer, Elderly, Survival

Posted Date: April 13th, 2021

DOI: https://doi.org/10.21203/rs.3.rs-410084/v1

License: (c) (i) This work is licensed under a Creative Commons Attribution 4.0 International License. Read Full License

Version of Record: A version of this preprint was published at World Journal of Surgical Oncology on August 11th, 2021. See the published version at https://doi.org/10.1186/s12957-021-02348-6. 


\section{Abstract}

Background: Only 50-70\% elderly colon cancer patients could complete a six-month postoperative chemotherapy. It is unknown whether a shorter duration of postoperative capecitabine-alone chemotherapy would not compromise the survival. We thus conducted this study to analyze the association between postoperative chemotherapy duration of capecitabine-alone regimen and cancer specific survival (CSS) in the surgery treated elderly colon cancer patients.

Methods: We performed a retrospective cohort study in surgery treated stage III and high-risk stage II colon cancer patients aged $>=70$ from two medical centers. Cox proportional hazard regression models were utilized to calculate crude and adjusted hazard ratios (HRs). The non-linear relationship between postoperative chemotherapy duration and CSS was analyzed through the restricted cubic spline regression analysis, and the threshold effect was calculated by the two-piece-wise Cox proportional hazard model.

Results: A total of 1,217 surgery treated colon cancer patients between August 1, 2013 and September 1, 2019 were reviewed and 257 stage III and high-risk stage II patients aged >= 70 were finally enrolled. Postoperative chemotherapy with capecitabine was delivered in 114 patients and 143 cases only received surgery. The capecitabine duration was generally associated with a $11 \%$ risk decrease in death $(H R=0.89$, $95 \%$ confidence interval (Cl) 0.82 - 0.96). Non-linearity exploration suggested a threshold effect of capecitabine duration on CSS in stage III disease. The HR for death was 0.79 (95\% Cl: 0.68 - 0.92) with duration <= 16 (week) while 1.34 (95\% Cl: 0.91 - 1.97) with duration > 16 (week).

Conclusions: The postoperative capecitabine duration was significantly associated with CSS in elderly colon cancer. However, a threshold effect of capecitabine duration on survival may suggest a short-term chemotherapy could be an alternative for the conventional six-month regimen in the elderly stage III colon cancer.

\section{Introduction}

Globally, colorectal cancer (CRC) is one of the five most common causes of cancer death (1). Patients with stage III and high-risk stage II colon cancer have a risk of recurrence over $30 \%$ after surgery $(2,3)$. Postoperative chemotherapy has been clearly proven to decrease the recurrence risk and increase survival in patients with high-risk stage II and stage III colon cancer (4). For several decades, a six-month chemotherapy regimen has been the standard postoperative treatment in colon cancer $(4,5)$. However, recent studies from International Duration Evaluation of Adjuvant Therapy collaboration (IDEA) suggested noninferiority of shorter duration (3 months) of chemotherapy, as compared with 6 months, was confirmed in most colon cancer patients with CAPOX (Oxaliplatin plus capecitabine) regimen, but not in those with FOLFOX (Oxaliplatin plus 5-fluorouracil/leucovorin) regimen $(6,7)$. The duration effect appeared dependent on the chemotherapy regimen and it seems that the use of capecitabine could shorten postoperative chemotherapy duration without compromising survival. 
So far, only a small fraction of fittest elderly was enrolled in randomized controlled trials (RCTs). Besides, the participants in the RCTs all received oxaliplatin-containing regimen, but it is still controversial to apply oxaliplatin-containing regimens for patients 70 years or older. A fluoropyrimidine alone regimen is still the recommended postoperative protocol for the elderly in clinical practice $(8,9)$. The limited number of elderly participants and the different recommended chemotherapy regimens for the elderly made it impossible to evaluate the effect of capecitabine duration on survival in the elderly from results of RCTs. Additionally, more than $30 \%-50 \%$ of patients could not complete the whole duration $(10,11)$. We were thus prompted to conduct this study to explore the association between the postoperative chemotherapy duration of capecitabine-alone regimen and cancer specific survival (CSS) in elderly colon cancer patients.

\section{Materials And Methods Study population}

The data of patients was collected from the electronic medical record system from Guizhou Province Cancer Hospital and Affiliated Hospital of Guizhou Medical University. Surgery treated patients aged $>$ or $=70$, with stage III and high-risk stage II colon cancer, were included. All patients enrolled were restaged based on the 8th edition of the Union for International Cancer Control / American Joint Cancer Committee TNM staging classification. Figure 1 shows the flow chart of enrollment. The inclusion criteria for this study were as follows: 1 . All patients enrolled were pathologically diagnosed as colonic adenocarcinoma; 2. They were aged $>=70$ years at the time of diagnosis and underwent radical surgery; 3 . They were staged as high-risk stage II (risk factors defined as tumor obstruction preoperatively, tumor perforation preoperatively, poorly differentiation, positive surgical margin, fewer than 12 lymph nodes detected, perineural infiltration, invasion of extramural venous or lymphatic vascular, or T4 disease) or stage III; 4 . The enrolled patients did not receive neoadjuvant treatment.

The local ethics committee of the Guizhou Province Cancer Hospital approved this study. Informed consent from enrolled patients was not required because this was a retrospective cohort study.

\section{Study design and variables}

This retrospective cohort study aimed to assess association between postoperative capecitabine duration and CSS in elderly colon cancer patients. The postoperative capecitabine duration was described as the time between the last cycle of chemotherapy accomplished and the first cycle of chemotherapy initiated. We obtained CSS as final outcome variable. CSS was defined as the interval from diagnosis to the date of cancer-attributable mortality. The Charlson comorbidity index $(\mathrm{CCl})$ has been proposed to evaluate the prognosis of CRC patients with comorbidities and frequently used in the Western countries (12-14). CCI was calculated using data regarding complications (15). We utilized clinical data from electronic medical record system to calculate $\mathrm{CCl}$ of each participant. Moreover, mismatch repair (MMR) status has been confirmed to be associated with response to fluoropyrimidines and recommended testing in CRC patients (16). Therefore, apart from demographic factors, $\mathrm{CCl}$ and clinicopathological features, we obtained MMR 
status as covariates. Finally, the variables used to establish multivariable adjusted models included: (1) continuous variable: age, carcinoma antigen 199 (CA199, obtained before surgery), carcinoembryonic antigen (CEA, obtained before surgery), $\mathrm{CCl}$ and (2) categorical variables: gender, $\mathrm{T}$ stage, $\mathrm{N}$ stage, histological type, and MMR status.

\section{Follow-up procedure}

Follow-up for enrolled patients through the outpatient visits was applied once every three months for the first 2 years, every 6 months for the next 3 years, and every 1 year thereafter. Complete physical examination, thoraco-abdominal CT, pelvic CT, serum CEA and CA199 assessment, and annual colonoscopy were monitored at each follow-up. The last date of follow-up was September 1, 2020. Information of follow-up was achieved by the second and third authors from electronic medical record system of Guizhou Province Cancer Hospital and Affiliated Hospital of Guizhou Medical University.

\section{Statistical analysis}

Continuous variables were described as mean \pm standard and categorical variables as frequency or percentage. Student's t-test (normal distribution), Mann-Whitney U test (skewed distribution), or $\chi 2$ (categorical variables) was adopted to investigate differences of characteristics of enrolled patients among various postoperative chemotherapy duration groups. Univariate Cox proportional hazard model and multivariate Cox proportional hazard model were applied to assess variates that may correlated with risk of death. Three models were constructed: model 1, not adjusted for any covariate; model 2, adjusted for gender, age; model 3, adjusted for covariates presented in Table 1. Stratified analyses were performed by Cox proportional hazard models. Cox proportional hazards regression model with cubic spline functions and smooth curve fitting were employed to explore nonlinear relationship between postoperative chemotherapy duration and CSS. If nonlinearity was detected, then the inflection point was calculated by recursive algorithm, and a two-piecewise Cox proportional hazard model was established on both sides of the inflection point. In the end, which model was more suitable for fitting the association between target independent variable and outcome variable was mainly determined by log likelihood ratio test. 
Table 1

Characteristics of enrolled patients

\begin{tabular}{|c|c|c|c|c|}
\hline \multirow[t]{2}{*}{ Characteristics } & \multicolumn{3}{|c|}{ Postoperative capecitabine duration } & \multirow[t]{2}{*}{ P-value } \\
\hline & 0 & $<=12$ weeks & $<=24$ weeks & \\
\hline No. of participants & 143 & 79 & 35 & \\
\hline Age (years), mean (SD) & $77.69(4.90)$ & $73.57(2.77)$ & 73.34 (2.99), & $<0.001$ \\
\hline$>=70,<75$ & $43(30.07 \%)$ & $46(58.23 \%)$ & $25(71.43 \%)$ & \\
\hline$>=75,<80$ & $43(30.07 \%)$ & $33(41.77 \%)$ & $8(22.86 \%)$ & \\
\hline$>=80$ & $57(39.86 \%)$ & $0(0.00 \%)$ & $2(5.71 \%)$ & \\
\hline Gender, N (\%) & & & & 0.421 \\
\hline Male & $85(59.44 \%)$ & $54(68.35 \%)$ & $22(62.86 \%)$ & \\
\hline Female & $58(40.56 \%)$ & $25(31.65 \%)$ & $13(37.14 \%)$ & \\
\hline T stage, N (\%) & & & & 0.177 \\
\hline $1-2$ & $8(5.59 \%)$ & $2(2.53 \%)$ & $1(2.86 \%)$ & \\
\hline 3 & $118(82.52 \%)$ & $68(86.08 \%)$ & $25(71.43 \%)$ & \\
\hline 4 & $17(11.89 \%)$ & $9(11.39 \%)$ & $9(25.71 \%)$ & \\
\hline N stage, $N(\%)$ & & & & 0.832 \\
\hline 0 & $74(51.75 \%)$ & 36 (45.57\%) & $18(51.43 \%)$ & \\
\hline 1 & 57 (39.86\%) & 33 (41.77\%) & $14(40.00 \%)$ & \\
\hline 2 & $12(8.39 \%)$ & 10 (12.66\%) & $3(8.57 \%)$ & \\
\hline Sage, N (\%) & & & & 0.664 \\
\hline II & 74 (51.75\%) & 36 (45.57\%) & 18 (51.43\%) & \\
\hline III & $69(48.25 \%)$ & 43 (54.43\%) & 17 (48.57\%) & \\
\hline Histological type & & & & 0.883 \\
\hline Common type & $122(85.31 \%)$ & 68 (86.08\%) & 31 (88.57\%) & \\
\hline Special type ${ }^{a}$, & $21(14.69 \%)$ & 11 (13.92\%) & $4(11.43 \%)$ & \\
\hline
\end{tabular}

Notes: aspecial type adenocarcinoma includes mucinous, signet ring cell, medullary, and undifferentiated variants.

Abbreviations: Abbreviations: CA, carcinoma antigen; CEA, carcinoembryonic antigen; MMR, mismatch repair; dMMR, mismatch repair deficiency; pMMR, proficient mismatch repair. 


\begin{tabular}{|c|c|c|c|c|}
\hline \multirow[t]{2}{*}{ Characteristics } & \multicolumn{3}{|c|}{ Postoperative capecitabine duration } & \multirow[t]{2}{*}{ P-value } \\
\hline & 0 & $<=12$ weeks & $<=24$ weeks & \\
\hline CA199, N (\%) & & & & 0.232 \\
\hline Normal & $122(85.31 \%)$ & $60(76.92 \%)$ & $27(77.14 \%)$ & \\
\hline Elevated & $21(14.69 \%)$ & $18(23.08 \%)$ & $8(22.86 \%)$ & \\
\hline CEA, N (\%) & & & & 0.830 \\
\hline Normal & $92(64.34 \%)$ & $48(60.76 \%)$ & $23(65.71 \%)$ & \\
\hline Elevated & $51(35.66 \%)$ & $31(39.24 \%)$ & $12(34.29 \%)$ & \\
\hline MMR status & & & & 0.021 \\
\hline dMMR & $15(10.49 \%)$ & $3(3.80 \%)$ & $2(5.71 \%)$ & \\
\hline pMMR & $106(74.13 \%)$ & $51(64.56 \%)$ & $28(80.00 \%)$ & \\
\hline Unknown & $22(15.38 \%)$ & $25(31.65 \%)$ & $5(14.29 \%)$ & \\
\hline Charlson comorbidity index & & & & $<0.001$ \\
\hline$<=4$ & $88(61.54 \%)$ & $75(94.94 \%)$ & $34(97.14 \%)$ & \\
\hline$>4$ & $55(38.46 \%)$ & $4(5.06 \%)$ & $1(2.86 \%)$ & \\
\hline \multicolumn{5}{|c|}{$\begin{array}{l}\text { Notes: a Special type adenocarcinoma includes mucinous, signet ring cell, medullary, and } \\
\text { undifferentiated variants. }\end{array}$} \\
\hline
\end{tabular}

We conducted a series of sensitivity analyses to guarantee the robustness of the data analysis. Firstly, due to the limitation of the Cox proportional-hazards model in addressing non-linearity, a generalized additive model was used to adjust covariates; we then compared its effect size with a fully-adjusted model. Secondly, we used different covariates as the stratification variables to observe the trend of HR among different subgroups and calculated the $\mathrm{P}$ for trend. All data analysis was performed with the statistical software packages R (http://www.R-project.org, The R Foundation) and EmpowerStats (http://www.empowerstats.com, X\&Y Solutions, Inc., Boston, MA, SUA). P values $<0.05$ (two-sided) was considered statistically significant.

\section{Results}

\section{Baseline characteristics of enrolled patients}


From August, 2013 to September, 2019, a total of 1,217 surgery treated colon cancer participants from Guizhou Province Cancer Hospital or Affiliated Hospital of Guizhou Medical University were retrospectively reviewed, and 257 elderly patients with stage III and high-risk stage II disease were enrolled into the final analysis. Postoperative chemotherapy with capecitabine-alone regimen was delivered in 114 $(114 / 257,44.36 \%)$ patients and 143 cases only received surgery. A total of 43 died of colon cancer (43/257, $16.73 \%)$ during follow-up, and 8 (8/257, 3.11\%) were lost to follow-up. The median follow-up time was 30.4 months. Table 1 describes baseline characteristics of enrolled patients across categories of chemotherapy duration. No statistically significant difference was found in measures except for age, MMR status, and $\mathrm{CCl}$ among different chemotherapy duration groups. Compared to patients receiving postoperative chemotherapy, those who only received operation were older. Moreover, patients without postoperative chemotherapy had higher $\mathrm{CCl}$ and MMR deficiency (dMMR) incidents.

\section{Association between postoperative chemotherapy duration and CSS}

The results of univariate analysis were shown in Table 2. Postoperative capecitabine duration was associated with a lower death risk (hazard ratio $(\mathrm{HR})=0.90,95 \% \mathrm{Cl}: 0.84-0.97$ ). Patients with postoperative capecitabine $<=12$ weeks or $>12$ weeks were both associated with a decrease in death, compared to those treated by surgery alone $(0.48,0.24-0.99$ and $0.09,0.01-0.67$ vs reference). While N2 $(5.31,2.42-11.64)$ vs reference), stage III $(2.45,1.26-4.78)$ vs stage II), and elevated CA199 $(2.04,1.04-$ 3.98 vs normal) were associated with a higher death risk. 
Table 2

Univariate analysis of enrolled patients

\begin{tabular}{|c|c|c|}
\hline \multirow[t]{2}{*}{ Variables } & \multirow[t]{2}{*}{ Mean \pm SD/N (\%) } & Cancer specific survival \\
\hline & & HR (95\% Cl), $\mathrm{P}$ value \\
\hline Postoperative capecitabine duration & $4.65 \pm 6.75$ & $0.90(0.84,0.97), 0.0043$ \\
\hline 0 & $143(55.64 \%)$ & 1.0 \\
\hline$<=12$ weeks & $79(30.74 \%)$ & $0.48(0.24,0.99), 0.046$ \\
\hline$<=24$ weeks & $35(13.62 \%)$ & $0.09(0.01,0.67), 0.018$ \\
\hline \multicolumn{3}{|l|}{ Gender } \\
\hline Male & $161(62.65 \%)$ & 1.0 \\
\hline Female & $96(37.35 \%)$ & $1.12(0.60,2.08), 0.73$ \\
\hline Age (years) & $75.83 \pm 4.60$ & $1.06(0.99,1.12), 0.080$ \\
\hline$>=70,<75$ & $114(44.36 \%)$ & 1.0 \\
\hline$>=75,<80$ & $84(32.68 \%)$ & $1.57(0.77,3.19), 0.21$ \\
\hline$>=80$ & $59(22.96 \%)$ & $1.79(0.83,3.87), 0.14$ \\
\hline \multicolumn{3}{|l|}{ T stage } \\
\hline $1-2$ & $11(4.28 \%)$ & 1.0 \\
\hline 3 & $211(82.10 \%)$ & $1.30(0.31,5.44), 0.71$ \\
\hline 4 & $35(13.62 \%)$ & $1.13(0.23,5.62), 0.88$ \\
\hline \multicolumn{3}{|l|}{$\mathrm{N}$ stage } \\
\hline 0 & $128(49.81 \%)$ & 1.0 \\
\hline 1 & $104(40.47 \%)$ & $1.77(0.85,3.67), 0.13$ \\
\hline 2 & $25(9.73 \%)$ & $5.31(2.42,11.64),<0.0001$ \\
\hline \multicolumn{3}{|l|}{ Stage } \\
\hline II & $128(49.81 \%)$ & 1.0 \\
\hline III & $129(50.19 \%)$ & $2.45(1.26,4.78), 0.0083$ \\
\hline \multicolumn{3}{|l|}{ Histological type } \\
\hline Common type & 221 (85.99\%) & 1.0 \\
\hline Special type & $36(14.01 \%)$ & $1.69(0.83,3.44), 0.15$ \\
\hline CEA & & \\
\hline
\end{tabular}




\begin{tabular}{|lll|}
\hline Variables & Mean \pm SD/N (\%) & Cancer specific survival \\
\cline { 2 - 3 } & & HR $(95 \%$ Cl), P value \\
\hline Normal & $163(63.42 \%)$ & 1.0 \\
\hline Elevated & $94(36.58 \%)$ & $1.15(0.62,2.12), 0.66$ \\
\hline CA199 & & \\
\hline Normal & $209(81.64 \%)$ & 1.0 \\
\hline Elevated & $47(18.36 \%)$ & $2.04(1.04,3.98), 0.037$ \\
\hline Charlson comorbidity index & & 1.0 \\
\hline$<=4$ & $197(76.65 \%)$ & $1.05(0.50,2.19), 0.90$ \\
\hline$>4$ & $60(23.35 \%)$ & \\
\hline MMR status & & 1.0 \\
\hline dMMR & $20(7.78 \%)$ & $0.51(0.21,1.23), 0.1338$ \\
\hline pMMR & $185(71.98 \%)$ & $0.63(0.22,1.76), 0.3769$ \\
\hline Unknown & $52(20.23 \%)$ & \\
\hline
\end{tabular}

As reported in Table 3, three models were used to analyze the independent association of postoperative capecitabine duration on CSS. In the unadjusted model, 0.90 (HR) demonstrated that capecitabine duration was associated with a $10 \%$ decrease in risk of death $(0.90,0.84-0.97)$. In the adjust I model, after adjusting for gender and age, the negative relationship was still robust $(0.90,0.84-0.97)$. In the adjusted II model, we adjusted for gender, age, stage, CEA (elevated, normal), CA199 (elevated, normal), $\mathrm{CCl}(<=4,>4)$, histological type and MMR status as confounding factors. After adjusting for confounding factors, capecitabine duration was associated with a $11 \%$ decrease in the risk of death $(0.89,0.82-0.96)$. Then postoperative capecitabine duration was transformed from continuous variable to categorical variable for sensitivity analysis. The results of postoperative capecitabine duration as categorical variables were consistent with the results when capecitabine duration was a continuous variable. 
Table 3

Results of multivariable analysis

\begin{tabular}{|c|c|c|c|}
\hline & Non-adjusted $^{a}$ & Adjust $\mathrm{I}^{\mathrm{b}}$ & Adjust $^{c}$ \\
\hline & HR (95\% Cl), P value & HR (95\% Cl), P value & HR ( $95 \% \mathrm{Cl})$, P value \\
\hline $\begin{array}{l}\text { Postoperative capecitabine } \\
\text { duration }\end{array}$ & $\begin{array}{l}0.90(0.84,0.97) \\
0.0043\end{array}$ & $\begin{array}{l}0.90(0.84,0.97) \\
0.0076\end{array}$ & $\begin{array}{l}0.89(0.82,0.96) \\
0.0035\end{array}$ \\
\hline 0 & 1.0 & 1.0 & 1.0 \\
\hline$<=12$ weeks & $\begin{array}{l}0.48(0.24,0.99) \\
0.046\end{array}$ & $\begin{array}{l}0.46(0.21,0.98) \\
0.045\end{array}$ & $\begin{array}{l}0.37(0.17,0.82), \\
0.015\end{array}$ \\
\hline$<=24$ weeks & $\begin{array}{l}0.09(0.01,0.67) \\
0.018\end{array}$ & $\begin{array}{l}0.09(0.01,0.69) \\
0.020\end{array}$ & $\begin{array}{l}0.07(0.01,0.56) \\
0.012\end{array}$ \\
\hline
\end{tabular}

Notes: ${ }^{a}$ Non-adjusted model adjusted for: None

${ }^{\mathrm{b}}$ Adjust I adjust for: Gender; Age.

CAdjust II adjust for: Gender; Age; Stage; CEA; CA199; CCl; Histological type and MMR status.

Abbreviations: HR: hazard ratio; Cl: confidence interval; CA: carcinoma antigen; CEA: carcinoembryonic antigen; MMR, mismatch repair; CCl: Charlson comorbidity index.

\section{Sensitivity analysis}

For sensitivity analysis, we first set up model II which adjusted for gender, age, stage, CEA (elevated, normal), CA199 (elevated, normal), $\mathrm{CCl}(<=4,>4)$, histological type and MMR status (Table 3$)$. The results were consistent with those of crude mode and model I. We further used different covariates as the stratification variables to observe the trend of HR among different subgroups (Fig. 2). The core results were consistent with our initial cohort. The tests for interactions were not statistically significant for age, gender, stage, histological type, CEA, CA199 or MMR status $(P>0.05)$.

\section{Non-linear relationship of postoperative chemotherapy duration with CSS in stage III patients}

In our study, the nonlinearity of postoperative capecitabine duration on CSS was observed in stage III (Fig. 3a, $\mathrm{P}$ for nonlinearity $=0.003)$, but not in high-risk stage II ( $\mathrm{P}$ for nonlinearity $=0.24)$. After adjusting for gender, age, T stage, CA199, CEA, histological type, CCI and MMR status, smooth curve analysis and Cox proportional hazards regression model with cubic spline functions indicated that the relationship between the postoperative capecitabine duration and CSS was still non-linear and presented as L-shape in stage III elderly patients (Fig. 3b, P for nonlinearity $=0.01$ ). Both Cox proportional hazard model and two-piecewise Cox proportional hazard model were applied to fit the association. We selected the best fit model according to $\mathrm{P}$ for log likelihood ratio test. Because the $\mathrm{P}$ for log likelihood ratio test was $<0.05$, we selected the two-piecewise Cox proportional hazard model for fitting the association between 
postoperative capecitabine duration and CSS in stage III elderly patients. The inflection point was calculated as 16 by using the recursive algorithm and two-piecewise Cox proportional hazard model (Table 4). The HR for the death was 0.79 (95\% Cl: 0.68-0.92) with postoperative capecitabine duration < $=16$ (week) while 1.34 (95\% Cl: 0.91-1.97) with duration > 16 (week). However, in high-risk stage II patients, we didn't discover this non-linear association.

Table 4

Results of the two-piecewise linear regression model in stage III elderly patients

\begin{tabular}{|c|c|}
\hline & HR (95\% Cl), P value ${ }^{a}$ \\
\hline Postoperative capecitabine duration & $0.86(0.78,0.96), 0.0046$ \\
\hline \multicolumn{2}{|l|}{ Inflection point of duration } \\
\hline$<16$ & $0.79(0.68,0.92), 0.0027$ \\
\hline$>=16$ & $1.34(0.91,1.97), 0.13$ \\
\hline P for log likelihood ratio test & 0.044 \\
\hline \multicolumn{2}{|c|}{ Notes: ${ }^{a}$ Adjust for: Gender, Age, T stage, CA199, CEA, Histological type, CCI and MMR. } \\
\hline
\end{tabular}

\section{Discussion}

In this retrospective cohort study, we investigated the association of postoperative chemotherapy duration of capecitabine-alone regimen to CSS for surgery treated elderly colon cancer patients. In general, the postoperative capecitabine duration was associated with a $11 \%$ decrease of death risk in the elderly, which was consistent with the findings mainly obtained from younger patients $(17,18)$. Further analyses in the stage III group unveiled some novel findings. Non-linearity exploration suggested that a Lshape relationship between the postoperative capecitabine duration and CSS. After adjusting potential confounding factors, the non-linear relationship between capecitabine duration and CSS was still significant (Fig. 3b, Table 4). The HR for the death was 0.79 (95\% Cl: 0.68-0.92) with chemotherapy duration $<=16$ (week) while 1.34 (95\% Cl: 0.91-1.97) with duration > 16 (week).

The duration effect appeared dependent on the use of capecitabine since patients with pT4 or pN2 tumor were still recommended with 6-months FOLFOX regimen, while CAPOX duration could be shorten to 3 months regardless of high-risk factors (17). Although capecitabine-alone regimen remains to be one of the recommended postoperative protocol for the elderly, studies reported less than $50 \%-70 \%$ of patients could complete the whole duration. Our study found that in stage III elderly colon cancer patients, the effect of capecitabine duration on CSS changed at the turning point of duration as 16 weeks. That is, after 16-week use of capecitabine, the declining trend of death risk as increasing in chemotherapy duration disappeared. To the best of our knowledge, this study firstly demonstrated the threshold effect 
of postoperative capecitabine duration on CSS in the elderly colon cancer. A short-term duration of capecitabine chemotherapy may be an alternative for the conventional six-month postoperative chemotherapy regimen in the elderly stage III colon cancer.

The threshold effect of capecitabine duration on survival which we discovered in stage III group was not detected in stage II group. The prognosis of stage II colon cancer varies greatly according to different risk factors. The 5-year survival rate of patients with stage IIB/IIC (T4) colon cancer was significantly lower than that of patients with stage IIIA (19). In addition, the prognosis of stage II patients with residual tumor was also worse than that of stage III patients, and the incidence of postoperative tumor residual in IIB/IIC stage was significantly higher than that in stage IIIA (20). Therefore, patients with certain high-risk factors in stage II may need longer capecitabine duration. A multicenter large cohort study may be needed to identify the effect of postoperative capecitabine duration on survival in terms of different high-risk factors in stage II.

Despite the International Society of Geriatric Oncology (SIOG) has recommended postoperative chemotherapy for elderly CRC patients, they tend to receive postoperative chemotherapy less frequently than non-elderly patients. In our study, $42.2 \%$ with high-risk stage II and $46.5 \%$ with stage III disease received postoperative therapy. Similarly, previous studies reported the proportion of elderly patients who received postoperative chemotherapy ranged from $15-57 \%(14,21-23)$. These proportions were much lower than those in younger patients. Reasons for nontreatment and treatment interruptions in the elderly included comorbidities, side effects and patient choice (23). Although SIOG has recommended the performance of a comprehensive geriatric assessment (CGA) before cancer treatment (24), CGA is not frequently used in clinical practice in China. Considering the comorbidities and tolerance of side effects in the elderly may affect the postoperative chemotherapy duration and outcome, we utilized clinical data from electronic medical record system to calculate $\mathrm{CCl}$ and then adjusted $\mathrm{CCl}$ in following Cox proportional hazard models.

Our study has some strengths. To our knowledge, this is the first study to evaluate the role of postoperative capecitabine duration for the elderly patients with stage III and high-risk stage II colon cancer. We firstly reported a significant association between postoperative capecitabine duration and CSS in the surgery treated elderly colon cancer. Furthermore, a L-shape relationship between capecitabine duration and CSS in stage III elderly colon cancer was discovered, and the threshold effect of capecitabine duration on CSS in this group was found. Strict statistical adjustment was applied to minimize residual confounders in this study.

There are still some limitations included in our study. Firstly, we could not evaluate the effect of postoperative chemotherapy duration of 5-fluorouracil-alone or other regimens since the regimen applied for elderly colon cancer was capecitabine in both two medical centers. A multi-center study is needed to extract more conclusions. Secondly, bias could be caused by unmeasured confounders and residual, but the sensitivity analysis indicated that the association between postoperative chemotherapy duration and CSS was robust. Thirdly, dose reduction or significant delay was not reported. We have not found 
available published data to assess the influence of dose reductions or treatment delays on survival in postoperative chemotherapy yet. Actually, a trial is ongoing to provide related knowledge (25).

\section{Conclusions}

In conclusion, the postoperative capecitabine duration was significantly associated with a decrease of death risk in elderly stage III and high-risk stage II colon cancer. However, a threshold effect of capecitabine duration on survival was found with the turning point as 16 weeks. A short-term duration of capecitabine chemotherapy may be an alternative for the conventional six-month postoperative chemotherapy regimen in the elderly stage III colon cancer.

\section{Abbreviations}

CRC

colorectal cancer

IDEA

International Duration Evaluation of Adjuvant Therapy collaboration

\section{CAPOX}

oxaliplatin plus capecitabine

\section{FOLFOX}

oxaliplatin plus 5 -fluorouracil/leucovorin

\section{RCTs}

randomized controlled trials

\section{CSS}

cancer specific survival

$\mathrm{CCl}$

Charlson comorbidity index

\section{MMR}

mismatch repair

CA199

carcinoma antigen 199

\section{CEA}

carcinoembryonic antigen

HR

hazard ratio

Cl

confidence interval

dMMR

mismatch repair deficiency

SIOG 
International Society of Geriatric Oncology

CGA

comprehensive geriatric assessment

\section{Declarations}

\section{Acknowledgements}

We would like to thank Zhining Cai for his editorial assistance in the preparation of this paper.

\section{Funding:}

This work was supported by the Guizhou Science and Technology Plan Project under Grant [2018]5779; and Technology Plan Project of Guizhou Cancer Hospital under Grant YJ2019-021.

\section{Availability of data and materials}

The dataset supporting the conclusions of this article is included within the article.

\section{Ethics approval and consent to participate}

This study was approved by the Ethics Committee of Guizhou Cancer Hospital (approval number: FZ 2020-4-050).

\section{Competing interests}

The authors declare that they have no competing interests

\section{Consent for publication}

Not applicable.

\section{Authors' contributions}

WWC and WLW designed the study. WWC, HMD, GW, JC and WLW involved in the postoperative management. WWC, HMD, GW, JC and WLW contributed to the collection and analysis of the study data. WWC wrote the article. HMD, GW, JC and WLW revised the manuscript. All authors read and approved the final manuscript.

\section{References}

1. Bray F, Ferlay J, Soerjomataram I, Siegel RL, Torre LA, Jemal A. Global cancer statistics 2018: GLOBOCAN estimates of incidence and mortality worldwide for 36 cancers in 185 countries. Cancer J Clin. 2018;68(6):394-424. 
2. Hatano S, Ishida H, Ishibashi K, Kumamoto K, Haga N, Miura I. Identification of risk factors for recurrence in high-risk stage II colon cancer. International surgery. 2013;98(2):114-21.

3. Brenner H, Kloor M, Pox CP. Colorectal cancer. The Lancet. 2014;383(9927):1490-502.

4. André T, Boni C, Navarro M, Tabernero J, Hickish T, Topham C, et al. Improved overall survival with oxaliplatin, fluorouracil, and leucovorin as adjuvant treatment in stage II or III colon cancer in the MOSAIC trial. Journal of clinical oncology: official journal of the American Society of Clinical Oncology. 2009;27(19):3109-16.

5. Kuebler JP, Wieand HS, O'Connell MJ, Smith RE, Colangelo LH, Yothers G, et al. Oxaliplatin combined with weekly bolus fluorouracil and leucovorin as surgical adjuvant chemotherapy for stage II and III colon cancer: results from NSABP C-07. Journal of clinical oncology: official journal of the American Society of Clinical Oncology. 2007;25(16):2198-204.

6. André T, Meyerhardt J, Iveson T, Sobrero A, Yoshino T, Souglakos I, et al. Effect of duration of adjuvant chemotherapy for patients with stage III colon cancer (IDEA collaboration): final results from a prospective, pooled analysis of six randomised, phase 3 trials. The Lancet Oncology. 2020;21(12):1620-9.

7. Iveson T, Sobrero A, Yoshino T, Souglakos I, Ou F, Meyers J, et al. Duration of Adjuvant Doublet Chemotherapy (3 or 6 months) in Patients With High-Risk Stage II Colorectal Cancer. Journal of clinical oncology: official journal of the American Society of Clinical Oncology. 2021;39(6):631-41.

8. Glimelius B, Osterman E. Adjuvant Chemotherapy in Elderly Colorectal Cancer Patients. Cancers. 2020;12(8).

9. Salem ME, Yin J, Goldberg RM, Pederson LD, Wolmark N, Alberts SR, et al. Evaluation of the change of outcomes over a 10-year period in patients with stage III colon cancer: pooled analysis of 6501 patients treated with fluorouracil, leucovorin, and oxaliplatin in the ACCENT database. Annals of oncology: official journal of the European Society for Medical Oncology. 2020;31(4):480-6.

10. van Erning FN, Janssen-Heijnen ML, Creemers GJ, Pruijt JF, Maas HA, Lemmens VE. Recurrence-free and overall survival among elderly stage III colon cancer patients treated with CAPOX or capecitabine monotherapy. International journal of cancer. 2017;140(1):224-33.

11. Hall M, Krell D, Glynne-Jones R. Single-agent capecitabine use in patients age 70 or older with colorectal cancer. J Clin Oncol. 2015;33(3_suppl):764-.

12. Tong L, Ahn C, Symanski E, Lai D, Du XL. Effects of newly developed chemotherapy regimens, comorbidities, chemotherapy-related toxicities on the changing patterns of the leading causes of death in elderly patients with colorectal cancer. Annals of oncology: official journal of the European Society for Medical Oncology. 2014;25(6):1234-42.

13. Hines RB, Chatla C, Bumpers HL, Waterbor JW, McGwin G, Funkhouser E, et al. Predictive Capacity of Three Comorbidity Indices in Estimating Mortality After Surgery for Colon Cancer. J Clin Oncol. 2009;27(26):4339-45.

14. Yamano T, Yamauchi S, Kimura K, Babaya A, Hamanaka M, Kobayashi M, et al. Influence of age and comorbidity on prognosis and application of adjuvant chemotherapy in elderly Japanese patients 
with colorectal cancer: A retrospective multicentre study. European journal of cancer (Oxford, England: 1990). 2017;81:90-101.

15. Charlson ME, Pompei P, Ales KL, MacKenzie CR. A new method of classifying prognostic comorbidity in longitudinal studies: development and validation. Journal of chronic diseases. 1987;40(5):37383.

16. Sargent DJ, Marsoni S, Monges G, Thibodeau SN, Labianca R, Hamilton SR, et al. Defective mismatch repair as a predictive marker for lack of efficacy of fluorouracil-based adjuvant therapy in colon cancer. Journal of clinical oncology: official journal of the American Society of Clinical Oncology. 2010;28(20):3219-26.

17. Grothey A, Sobrero AF, Shields AF, Yoshino T, Paul J, Taieb J, et al. Duration of Adjuvant Chemotherapy for Stage III Colon Cancer. N Engl J Med. 2018;378(13):1177-88.

18. Iveson T, Sobrero AF, Yoshino T, Sougklakos I, Ou F-S, Meyers JP, et al. Prospective pooled analysis of four randomized trials investigating duration of adjuvant (adj) oxaliplatin-based therapy (3 vs 6 months $\{\mathrm{m}\})$ for patients (pts) with high-risk stage II colorectal cancer (CC). J Clin Oncol. 2019;37(15_suppl):3501-.

19. Kim M, Jeong S, Choi S, Ryoo S, Park J, Park K, et al. Survival paradox between stage IIB/C (T4N0) and stage IIIA (T1-2N1) colon cancer. Ann Surg Oncol. 2015;22(2):505-12.

20. Chu Q, Zhou M, Medeiros K, Peddi P. Positive surgical margins contribute to the survival paradox between patients with stage IIB/C (T4N0) and stage IIIA (T1-2N1, T1N2a) colon cancer. Surgery. 2016;160(5):1333-43.

21. Doat S, Thiébaut A, Samson S, Ricordeau P, Guillemot D, Mitry E. Elderly patients with colorectal cancer: treatment modalities and survival in France. National data from the ThInDiT cohort study. European journal of cancer (Oxford, England: 1990). 2014;50(7):1276-83.

22. Kurtz JE, Heitz D, Serra S, Brigand C, Juif V, Podelski V, et al. Adjuvant chemotherapy in elderly patients with colorectal cancer. A retrospective analysis of the implementation of tumor board recommendations in a single institution. Crit Rev Oncol Hematol. 2010;74(3):211-7.

23. Ko JJ, Kennecke HF, Lim HJ, Renouf DJ, Gill S, Woods R, et al. Reasons for Underuse of Adjuvant Chemotherapy in Elderly Patients With Stage III Colon Cancer. Clin Colorectal Cancer. 2016;15(2):179-85.

24. Papamichael D, Audisio RA, Glimelius B, de Gramont A, Glynne-Jones R, Haller D, et al. Treatment of colorectal cancer in older patients: International Society of Geriatric Oncology (SIOG) consensus recommendations 2013. Annals of oncology: official journal of the European Society for Medical Oncology. 2015;26(3):463-76.

25. Lund CM, Vistisen KK, Dehlendorff C, Rønholt F, Johansen JS, Nielsen DL. The effect of geriatric intervention in frail elderly patients receiving chemotherapy for colorectal cancer: a randomized trial (GERICO). BMC Cancer. 2017;17(1):448.

\section{Figures}


Colon cancer patients from August 1, 2013 to

September 1, 2019

$(\mathrm{N}=1217)$
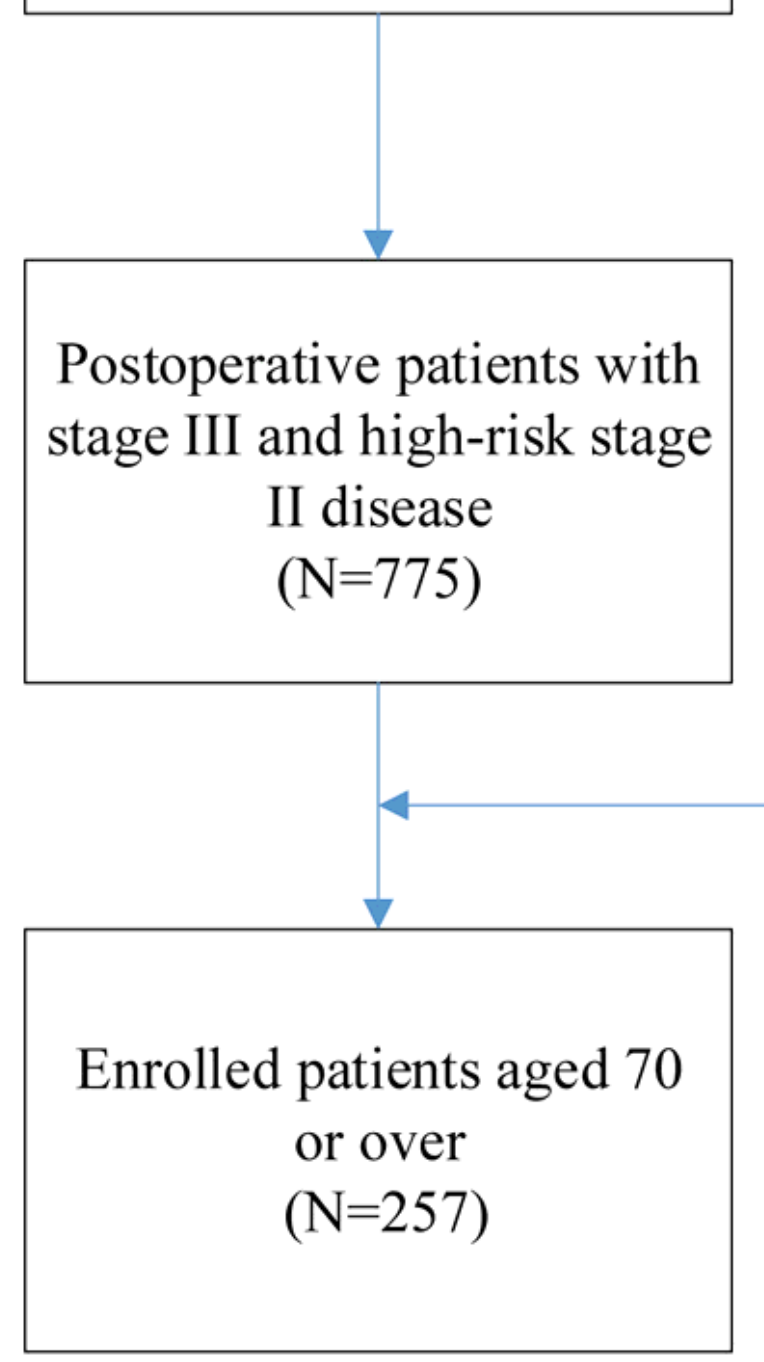

\section{Exclusion:}

1、 Patients aged $<70$

2、Patients with neoadjuvant chemotherapy 3、 Patients without radical surgery

\section{Figure 1}

Flow chart of enrollment. 
Stratified analysis

Gender

Female

Male

Age

$<75$

$>=75$

Stage

II

III

Histological type

Common

Special

CEA

Normal

Elevated

CA199

Normal

Elevated

MMR

dMMR

pMMR

Unknown
$\mathrm{N}$

161

96

114

143

128

129

221

36

163

94

209

47

20

185

52
HR P for interaction

0.34

0.92

0.84

0.69

0.35

0.92

0.79

0.91

0.89

0.45

0.89

0.95

0.075

0.94

0.80

0.22

0.92

0.83

0.36

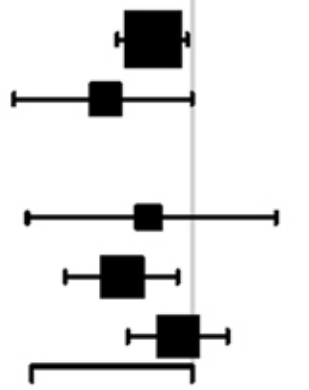

0.91

0.86

0.97

Figure 2

Subgroup analyses by gender, age, stage, histological type, CEA, CA199, MMR status in stage III and highrisk stage II colon cancer. 

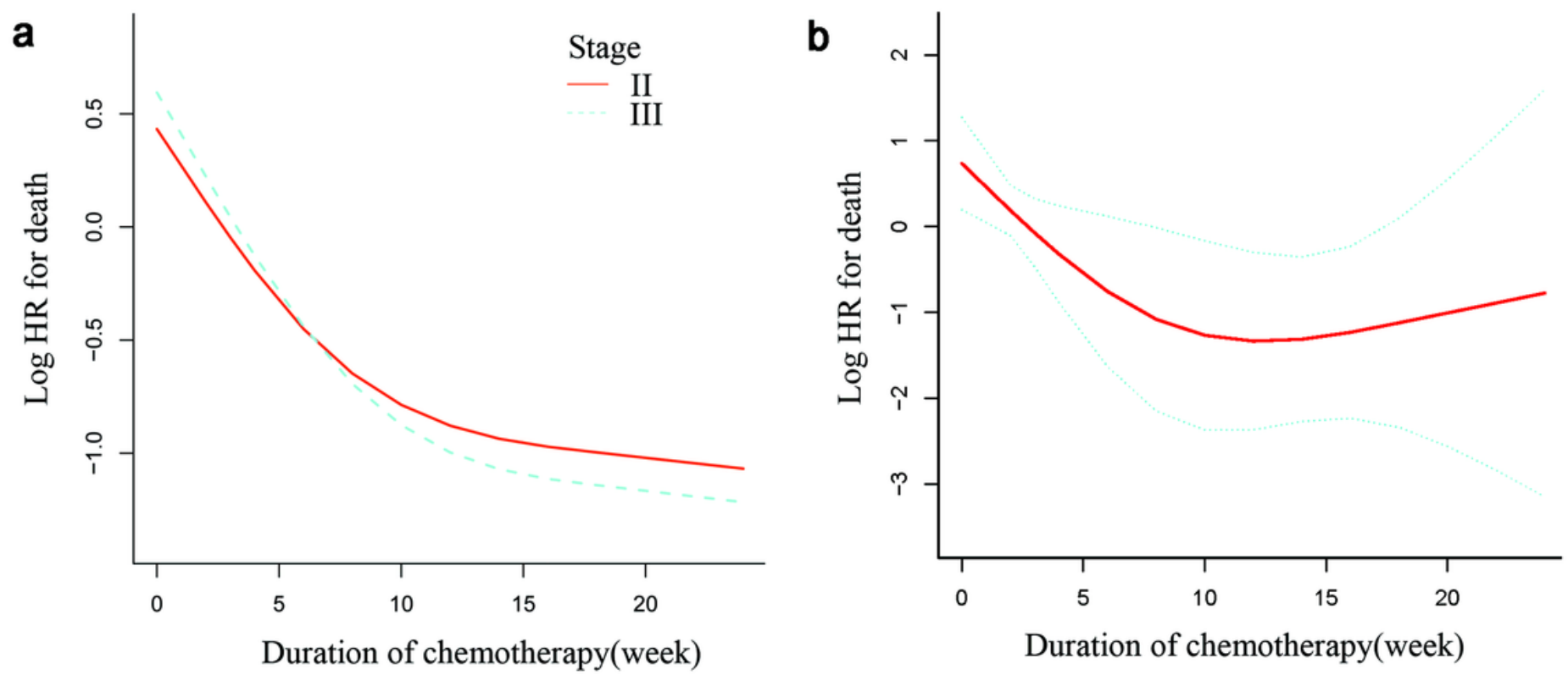

\section{Figure 3}

(A) The non-linear relationship between postoperative capecitabine duration and CSS was observed in stage III disease (P for nonlinearity $=0.003)$, but not in stage II disease ( $P$ for nonlinearity $=0.24)$. $(B)$ The red curved line shows a L-shape relationship between the capecitabine duration and the risk of death in stage III elderly colon cancer after adjusting for gender, age, T stage, CA199, CEA, histological type, CCI and MMR status. The area between the two blue dotted lines is expressed as a $95 \% \mathrm{Cl}$. The HR for the death was $0.79(95 \% \mathrm{Cl}: 0.68$ - 0.92) with postoperative capecitabine duration $<=16$ (week) while 1.34 $(95 \% \mathrm{Cl}: 0.91-1.97)$ with duration > 16 (week). 